\title{
Influência do tempo de contagem na determinação da densidade de nêutrons
}

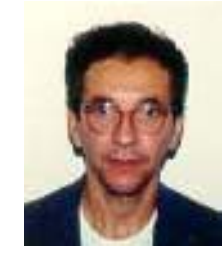

\author{
Antônio R. S. de Andrade ${ }^{1}$, Sidnei O. Jadoski², Hugo C. Guerra ${ }^{3}$ \& Ivan A. Guerrini ${ }^{4}$
}

\author{
1 FCA/UNESP. Campus de Rubião Junior, Botucatu, SP. E-mail: arsa@fca.unesp.br (Foto) \\ 2 UNESP. Campus de Rubião Junior, Botucatu, SP. E-mail: jadoski@fca.unesp.br \\ ${ }^{3}$ DEAg/CCT/UFPB, Av. Aprígio Veloso, 882, Bodocongó, CEP 58109-970, Campina Grande, PB. Fone: (83) 310-1285. \\ E-mail: hugo@deag.ufpb.br \\ 4 UNESP. Campus de Rubião Junior, Botucatu, SP. E-mail: gerrini@ibb.unesp.br
}

Protocolo $007-22 / 1 / 2001$

\begin{abstract}
Resumo: Com o objetivo de se estudar a influência do tempo de contagem na determinação da densidade de nêutrons moderados com uma sonda de nêutrons, num Latossolo localizado na área experimental do Departamento de Engenharia Rural, da Faculdade de Ciências Agronômicas, Universidade Estadual Paulista, Campus de Botucatu, SP, coletaram-se dados no interior de uma estufa de polietileno, a três profundidades no solo, 15, 30 e $45 \mathrm{~cm}$, com tempos de contagem de $1,4,16,32,64,128$ e 256 s, com cinco repetições. Para avaliação da variabilidade entre as leituras, utilizaram-se métodos estatísticos descritivos: variância, desvio-padrão, valores de amplitude e coeficiente de variação. Para se determinar a influência do tempo de contagem no valor das leituras, utilizou-se o teste $\mathrm{F}$ e comparação entre médias através do teste de Tukey. Com exceção da profundidade de $45 \mathrm{~cm}$, observou-se pequena variabilidade entre as leituras obtidas para cada tempo de contagem e uma significante variação entre as médias das leituras obtidas com curtos (1 e 4 s) e longos (tempos maiores de $16 \mathrm{~s}$ ) tempos de contagem. O uso de longos tempos de contagem (maiores de 32 s) não ocasiona diferença significativa na magnitude das leituras.
\end{abstract}

Palavras-chave: sonda de nêutrons, tempo de contagem, distribuição de Poisson

\section{Influence of the counting time on neutron density determination}

\begin{abstract}
To study the influence of the neutron probe counting time on neutron density determination a study was carried out on a Latossol, under greenhouse conditions, at the Experimental Station of the Rural Engineering Department, UNESP in Botucatu-SP. Neutron probe data were collected, with five replicates, at three soil depths $(15,30$ and $45 \mathrm{~cm})$ with counting times of $1,4,16,32,128$ and $256 \mathrm{~s}$. To evaluate the variability among replicates, conventional statistics parameters were determined : variance, standard deviation, amplitude and coefficient of variation; to determine the influence of the counting time on neutron density determination, analyses of variance ( $F$ test) and comparisons of means (Tukey Test) were also conducted. The results showed small variability among readings for each counting time and significant variation among the reading means for the short ( 1 and $4 \mathrm{~s}$ ) and long (longer than $16 \mathrm{~s})$, counting times with the exception of the $45 \mathrm{~cm}$ soil depth. The use of long counting times (longer than $32 \mathrm{~s}$ ) does not cause any significant difference on the readings magnitude.
\end{abstract}

Key words: Neutron probe, radiation counting time, Poisson distribution

\section{INTRODUÇÃO}

A sonda de nêutrons é um instrumento utilizado para a determinação do conteúdo de água do solo. As principais vantagens do método são: permitir a obtenção de valores de umidade do solo no mesmo ponto e a qualquer tempo sem deformar o solo, ser rápido e preciso, apresentando a possibilidade de alta periodicidade de medição, de fácil manejo e relativamente barato e poder ser conectado a registradores, possibilitando leituras contínuas no campo. (Torres \& Gonzáles, 1993). Sua capacidade de trabalho estende-se a solos bem mais secos, onde os tensiômetros não podem ser utilizados.

O principio da metodologia de determinação da umidade do solo com sonda de nêutrons consiste, segundo Guerra (2000) em colocar uma fonte radioativa a uma profundidade desejada no solo (através de um tubo de acesso, de aço ou alumínio, inserido verticalmente no solo) em que os nêutrons rápidos são emitidos, penetrando radialmente no solo, onde se encontram vários núcleos atômicos com os quais colidem elasticamente, perdendo energia cinética. Esta perda é máxima quando o nêutron bate com uma partícula de massa similar à sua. Na prática, tem-se 
encontrado que a moderação de nêutrons rápidos no solo é proporcional ao conteúdo de hidrogênio no solo (com núcleos de massa similar aos nêutrons). Assim, quando os nêutrons rápidos colidem com os núcleos de hidrogênio da água do solo, esses nêutrons perdem sua máxima energia formando, ao redor da fonte radioativa, uma nuvem de nêutrons moderados (ou termalizados) os quais são captados pelo detector, geralmente constituído de $\mathrm{BF}_{3}$ (Trifluoreto de boro) e assim, quando um nêutron moderado ou lento encontra ${ }_{5}^{10} \mathrm{~B}$ e é absorvido, uma partícula de He é emitida, criando um pulso elétrico. Este pulso, por sua vez, é enviado, através de um cabo, ao medidor, onde uma leitura é registrada. Quanto mais hidrogênio no solo (mais água) mais nêutrons rápidos são moderados, maior o número de pulsos enviados ao medidor e maior a leitura.

Os erros cometidos na obtenção de leituras provenientes de uma fonte emissora de nêutrons são, comumente, de dois tipos: erro devido à emissão ao acaso de nêutrons e erro associado com o equipamento utilizado. Para qualquer tempo, a contagem de nêutrons segue a distribuição de Poisson com média igual a $n$ e desvio-padrão ${ }^{1 / 2}$ (Gardner, 1985). Se um número adequado de medições é feito e a informação plotada numa curva de distribuição de desvios com respeito a média, se encontrará que a contagem segue a familiar curva de distribuição normal, a qual para a maioria das medições nucleares é uma adequada aproximação da distribuição de Poisson (Wang and Willis, 1965). Em geral, as contagens são feitas para se obter aproximações equivalentes a um desvio padrão de $1 \%$.

As leituras utilizadas para determinar a umidade do solo utilizando-se a sonda de nêutrons, podem ser realizadas em diferentes tempos de acionamento do monitor de contagem dos nêutrons moderados, a partir de um ajuste do menu de comando do equipamento.

Apesar da sonda de nêutrons já estar em uso por mais de três décadas, ainda existe discussão ou controvérsia acerca do tempo de contagem mais adequado. Chawla \& Tripathi (1987); Carneiro \& De Jong (1989) e Encarnação et al. (1991) apresentam várias metodologias de campo e de laboratório, para a calibração de sondas e determinação da umidade do solo; no entanto, não é levada em conta a influência do tempo; já Beltrame \& Taylor (1980), Falleiros et al. (1994) e Marciano (1995), aconselharam leituras com sonda de nêutrons usando tempos de contagem longos, no sentido de se obter valores de umidade mais próximos do real. Horiushi et al. (2000) salientam que o tempo de contagem é, juntamente com a natureza e a densidade do solo, o principal determinante da precisão das determinações do teor de água do solo com o uso da sonda de nêutrons.

O presente trabalho teve como objetivo estudar a influência do tempo de contagem na magnitude das leituras e sua variabilidade dentro de cada tempo de contagem.

\section{MATERIAL E MÉTODOS}

Este trabalho foi desenvolvido numa estufa de polietileno transparente de baixa densidade, com dimensões de 7,5 m de largura por $28 \mathrm{~m}$ de comprimento, instalada na área experimental do Departamento de Engenharia Rural, da Faculdade de Ciências Agronômicas, Universidade Estadual Paulista,
Campus de Botucatu, SP. O solo, no interior da estufa, sem cultivo, é classificado como Terra Estruturada Latossólica.

A sonda de nêutrons utilizada foi uma sonda CPN modelo 503 DR Hidroprobe (50 mCi Am-241/Be) introduzida no solo através de tubos de acesso, de alumínio, com $48 \mathrm{~mm}$ e $50 \mathrm{~mm}$ de diâmetro interno e externo, respectivamente. Os tempos de contagem estudados foram de 1, 4, 16, 32, 64, 128 e $256 \mathrm{~s}$. As leituras foram realizadas com cinco repetições nas profundidades de 15,30 e $45 \mathrm{~cm}$, quando o conteúdo de água do solo era de 24,47; 24,13 e 24,45\%, respectivamente. Para se avaliar a variabilidade entre as leituras, utilizaram-se métodos estatísticos clássicos: variância, desvio-padrão, valores de amplitude e coeficiente de variação e, para se identificar a distribuição teórica mais representativa do comportamento dos dados observados, usaram-se os coeficientes de assimetria e curtose. O delineamento estatístico utilizado para determinação da influência do tempo de contagem no valor das leituras foi inteiramente casualizado, com 7 tratamentos (tempos de contagem) e 5 repetições, para cada profundidade em estudo. As médias dos tratamentos foram comparadas utilizando-se o teste Tukey a nível de significância de 5\%, conforme Gomes (1987).

\section{RESULTADOS E DISCUSSÃO}

A partir dos valores de leitura (taxas de contagem em cpm) obtidos com a sonda de nêutrons com os sete tempos de contagem $(1,4,16,32,64,128 \mathrm{e} 256 \mathrm{~s})$ para as três profundidades do solo $(15,30$ e $45 \mathrm{~cm})$ foram obtidos os momentos estatísticos apresentados na Tabela 1, os quais resumem o comportamento geral dos dados. Os baixos coeficientes de variação, desvios padrões e amplitudes observados para cada tempo de contagem e profundidade indicam, em geral, pequena variabilidade entre as repetições. Com exceção dos tempos de contagem de 1 e $4 \mathrm{~s}$, os desvios padrões, indicadores da exatidão de qualquer medida individual, foram inferiores a $1 \%$, exatidão considerada aceitável nesse tipo de medições, segundo Gardner (1985). Igualmente, com exceção dos tempos de contagem de 1 e $4 \mathrm{~s}$, todos os coeficientes de variação foram inferiores a 1 , o que segundo Havercamp et al. (1984) revela pequena ou insignificante variabilidade das leituras. Para as leituras realizadas aos 1 e $4 \mathrm{~s}$, os coeficientes de variação foram superiores a 1 revelando, assim, inadequada variabilidade dos dados. Observa-se, também, aumento da variabilidade dos valores de leitura somente para o tempo de contagem de $1 \mathrm{~s}$, com o acréscimo da profundidade. Esta variabilidade se deve, provavelmente, à heterogeneidade das características físicas do solo, como textura e densidade, que influenciam diretamente nas leituras obtidas pela sonda. Outros erros durante a realização dos testes, embora em pequena escala, poderiam ser apontados como outras possíveis causas. Mishima \& Hibiki (1996) salientam que a ocorrência de diferenças de temperatura nos pontos de leitura com a sonda, podem afetar os resultados das determinações.

Coeficientes de assimetria praticamente nulos e coeficientes de curtose menores que 3 , induzem à aceitação da hipótese de normalidade, ou seja, como afirmado por Wang \& Willis (1965) é possível ajustar-se a contagem de nêutrons a uma distribuição normal (distribuição observada); confirma-se, com isto, que as 
Tabela 1. Momentos estatísticos dos valores de leitura da sonda de nêutrons (taxas de contagem em cpm) para os diferentes tempos de contagem, para as três profundidades de solo

\begin{tabular}{|c|c|c|c|c|c|c|c|}
\hline \multirow{2}{*}{ Estatística } & \multicolumn{7}{|c|}{ Tempo de Leitura (s) } \\
\hline & 1 & 4 & 16 & 32 & 64 & 128 & 256 \\
\hline & \multicolumn{7}{|c|}{ Profundidade $15 \mathrm{~cm}$} \\
\hline Média & 8473,60 & 8607,20 & 8723,40 & 8709,40 & 8664,00 & 8741,60 & 8733,20 \\
\hline Mediana & 8448,00 & 8668,00 & 8699,00 & 8720,00 & 8669,00 & 8742,00 & 8732,00 \\
\hline Variância & 47820,80 & 23947,20 & 7718,30 & 1894,30 & 1124,50 & 96,80 & 601,70 \\
\hline Desvio padrão & 218,68 & 154,75 & 87,85 & 43,52 & 33,53 & 9,84 & 24,53 \\
\hline Valor máximo & 8768,00 & 8740,00 & 8865,00 & 8745,00 & 8704,00 & 8753,00 & 8765,00 \\
\hline Valor mínimo & 8224,00 & 8384,00 & 8626,00 & 8635,00 & 8611,00 & 8729,00 & 8702,00 \\
\hline Amplitude total & 544,00 & 356,00 & 239,00 & 110,00 & 93,00 & 24,00 & 63,00 \\
\hline Coef. de variação & 2,58 & 1,80 & 1,01 & 0,50 & 0,39 & 0,11 & 0,28 \\
\hline Coef. de assimetria & 0,17 & $-0,40$ & 0,56 & $-0,85$ & $-0,45$ & $-0,08$ & 0,03 \\
\hline \multirow[t]{2}{*}{ Coef. de curtose } & 1,07 & 1,09 & 1,65 & 1,82 & 1,65 & 1,00 & 1,14 \\
\hline & \multicolumn{7}{|c|}{ Profundidade $30 \mathrm{~cm}$} \\
\hline Média & 11440,00 & 11004,80 & 11067,60 & 11083,40 & 11125,20 & 11145,20 & 11167,60 \\
\hline Mediana & 11504,00 & 11012,00 & 11074,00 & 11076,00 & 11118,00 & 11163,00 & 11155,00 \\
\hline Variância & 88192,00 & 2603,20 & 3777,80 & 4647,30 & 404,20 & 1314,70 & 651,80 \\
\hline Desvio padrão & 296,97 & 51,02 & 61,46 & 68,17 & 20,10 & 36,26 & 25,53 \\
\hline Valor máximo & 11856,00 & 11056,00 & 11143,00 & 11195,00 & 11153,00 & 11179,00 & 11211,00 \\
\hline Valor mínimo & 11136,00 & 10920,00 & 10987,00 & 11015,00 & 11101,00 & 11092,00 & 11149,00 \\
\hline Amplitude total & 720,00 & 136,00 & 156,00 & 180,00 & 52,00 & 87,00 & 62,00 \\
\hline Coef. de variação & 2,60 & 0,46 & 0,56 & 0,62 & 0,18 & 0,33 & 0,23 \\
\hline Coef. de assimetria & 0,19 & $-0,70$ & $-0,09$ & 0,64 & 0,20 & $-0,40$ & 0,84 \\
\hline \multirow[t]{2}{*}{ Coef. de curtose } & 1,13 & 1,74 & 1,10 & 1,66 & 1,18 & 1,14 & 1,76 \\
\hline & \multicolumn{7}{|c|}{ Profundidade $45 \mathrm{~cm}$} \\
\hline Média & 11309,60 & 11465,80 & 11587,80 & 11533,80 & 11498,60 & 11535,20 & 11525,00 \\
\hline Mediana & 11168,00 & 11384,00 & 11606,00 & 11503,00 & 11502,00 & 11529,00 & 11526,00 \\
\hline Variância & 95484,80 & 49883,20 & 3811,20 & 3619,70 & 506,30 & 884,20 & 431,50 \\
\hline Desvio padrão & 309,01 & 223,35 & 61,73 & 60,16 & 22,50 & 29,74 & 20,77 \\
\hline Valor máximo & 11696,00 & 11764,00 & 11642,00 & 11624,00 & 11523,00 & 11577,00 & 11551,00 \\
\hline Valor mínimo & 10960,00 & 11232,00 & 11493,00 & 11479,00 & 11473,00 & 11500,00 & 11495,00 \\
\hline Amplitude total & 736,00 & 532,00 & 149,00 & 145,00 & 50,00 & 77,00 & 56,00 \\
\hline Coef. de variação & 2,73 & 1,95 & 0,53 & 0,52 & 0,20 & 0,26 & 0,18 \\
\hline Coef. de assimetria & 0,17 & 0,27 & $-0,50$ & 0,48 & $-0,08$ & 0,22 & $-0,20$ \\
\hline Coef. de curtose & 0,94 & 0,99 & 1,31 & 1,21 & 0,84 & 1,21 & 1,37 \\
\hline
\end{tabular}

leituras médias para cada profundidade são ótimos indicadores do valor médio das leituras que, neste caso, poderão ser usados como valores representativos das profundidades do solo estudado. De acordo com os valores dos coeficientes de curtose obtidos, verifica-se que todos são inferiores a 3, o que significa que há uma disfunção dos dados em termos de média, maior que uma curva gaussiana (curva normal) ou seja, que a amostra dos dados para todas as profundidades estudadas é platicúrtica em relação à normal (mais pontiaguda) constatando-se, assim, menor variabilidade das leituras ao redor da média.

Os valores médios das leituras obtidas com a sonda de nêutrons, para os diferentes tempos de contagem e profundidades, são representados na Tabela 2 .

Pelos dados apresentados na Tabela 2, verifica-se que, com exceção da profundidade de $45 \mathrm{~cm}$, na qual não houve diferença significativa entre as médias das leituras para os diferentes tempos de contagem, nas outras duas profundidades $(15 \mathrm{e}$ $30 \mathrm{~cm}$ ) o tempo de contagem influenciou significativamente. $\mathrm{Na}$ profundidade de $15 \mathrm{~cm}$ embora não houvesse diferença significativa entre as leituras obtidas a 1 e $4 \mathrm{~s}$, a primeira foi significativamente diferente daquelas obtidas a tempos maiores de $4 \mathrm{~s}$; já para a profundidade de $30 \mathrm{~cm}$, verifica-se diferença significativa entre as médias obtidas a $1 \mathrm{~s}$ com os demais tempos de contagem. Por outro lado, os resultados do testes estatísticos
Tabela 2. Valores médios* das leituras obtidos sob diferentes tempos de contagem, em segundos, para as três profundidades

\begin{tabular}{cccc}
\hline $\begin{array}{c}\text { Tempo de } \\
\text { Contagem } \\
\text { (s) }\end{array}$ & \multicolumn{3}{c}{$\begin{array}{c}\text { Leitura para } \\
\text { Profundidade }(\mathrm{cm})\end{array}$} \\
\cline { 2 - 4 } 1 & $8473,60 \mathrm{a}$ & $11440,00 \mathrm{a}$ & $11309,60 \mathrm{a}$ \\
4 & $8607,20 \mathrm{ab}$ & $11004,80 \mathrm{~b}$ & $11465,80 \mathrm{a}$ \\
16 & $8723,40 \mathrm{~b}$ & $11067,60 \mathrm{~b}$ & $11587,80 \mathrm{a}$ \\
32 & $8709,40 \mathrm{~b}$ & $11083,40 \mathrm{~b}$ & $11533,80 \mathrm{a}$ \\
64 & $8664,00 \mathrm{ba}$ & $11125,20 \mathrm{~b}$ & $11498,60 \mathrm{a}$ \\
128 & $8741,60 \mathrm{~b}$ & $11145,20 \mathrm{~b}$ & $11535,20 \mathrm{a}$ \\
256 & $8733,20 \mathrm{~b}$ & $11167,60 \mathrm{~b}$ & $11525,00 \mathrm{a}$ \\
DMS 5\% & 218,76 & 241,72 & 208,21 \\
C. V. \% & 0,26 & 1,08 & 1,29 \\
Média total & 8664,62 & 11147,68 & 11493,31 \\
\hline * As médias seguidas de mesma letra, nas colunas, não diferem estatisticamente pelo teste Tukey, a nivel de 0,05
\end{tabular}
da profundidade

mostraram que o uso de tempos de contagem superiores a $16 \mathrm{~s}$ não influencia na magnitude da leitura e teoricamente na determinação do conteúdo de água do solo contradizendo, assim, resultados obtidos em trabalhos similares, nos quais pesquisadores aconselham leituras com sonda de nêutrons, usando tempos de contagem longo no sentido de se obter valores de umidade mais próximos do real (Beltrame \& Taylor, 1980; Falleiros et al., 1994; Marciano, 1995). 
Verificada a hipótese de normalidade dos dados através dos testes de assimetria e curtose, estimou-se o número de leituras necessárias para cada tempo de contagem, a nível de significância de 5\%, para uma variação de 5 a $30 \%$ em torno da estimativa da média.

A Figura 1 apresenta o número de leituras necessário para os diferentes tempos de contagem, em suas respectivas profundidades, a nível de 5\% de probabilidade, em função do erro percentual admitido em torno da média, calculado segundo metodologia de Costa Neto (1990). Considerando-se, por exemplo, uma variação de $5 \%$ a nível de significância de $5 \%$, os números de leituras requeridos para a profundidade de $15 \mathrm{~cm}$ seriam, aproximadamente, duas leituras para os tempos de contagem 1, 4 e $16 \mathrm{~s}$, e de uma leitura para tempos maiores de $16 \mathrm{~s}$. Para este mesmo nível de significância e uma variação de $10 \%$, o número requerido de leituras seria de uma para os tempos de contagem 1,4 e $16 \mathrm{~s}$ e permaneceria inalterado (uma leitura) para os tempos de 32 a $256 \mathrm{~s}$. Este exemplo mostra que uma redução de $5 \%$ na precisão da média, usando-se os tempos de contagens 1, 4 e $16 \mathrm{~s}$, proporcionaria uma redução de aproximadamente $50 \%$, em relação à necessidade de leituras a serem realizadas com a sonda; já para os tempos de contagem de 32 a 256 s, nenhuma alteração seria necessária no número

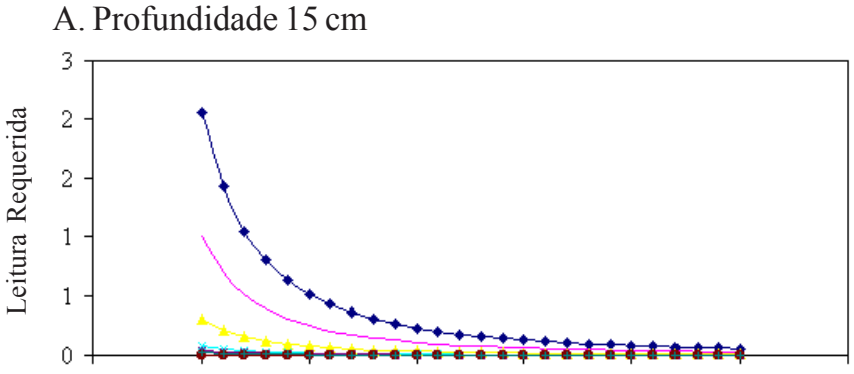

B. Profundidade $30 \mathrm{~cm}$

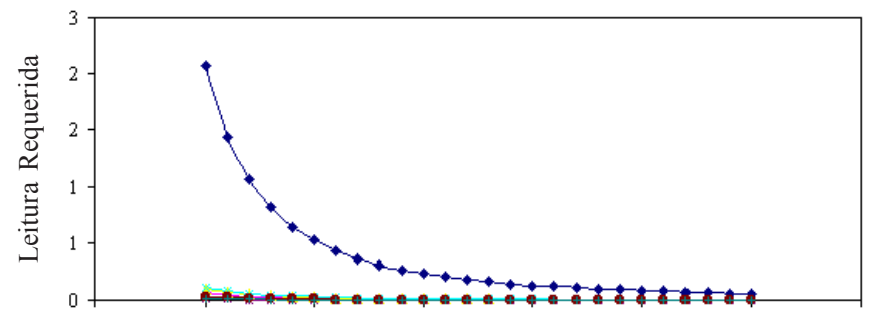

C. Profundidade $45 \mathrm{~cm}$

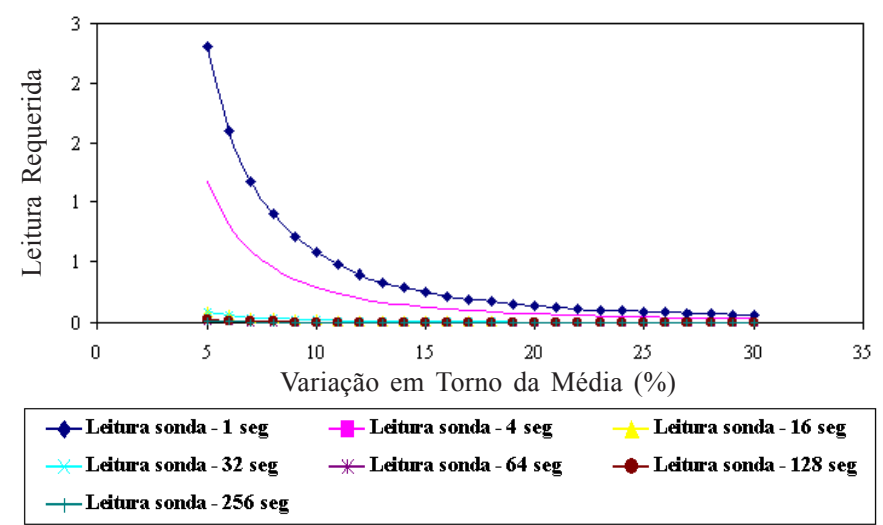

Figura 1. Número de leituras requerido para os diferentes tempos de contagem para as três profundidades, com 5 a $30 \%$ de precisão, à nível de $5 \%$ de probabilidade requerido de leituras. Para variações próximas da media maiores de $15 \%$, o tempo de contagem praticamente não afeta o número de leituras necessárias. Considerando-se razoável uma variação de $5 \%$ em torno da média, a melhor opção parece ser o uso de uma leitura aos $32 \mathrm{~s}$, tempo normalmente utilizado na prática.

A análise da Figura 1 permite, ainda, observar-se que o número requerido de leituras para cada tempo de contagem diminui a medida em que aumenta a profundidade, fato este associado à menor variabilidade das leituras com o aumento da profundidade, conforme mostrado através das medidas de variabilidade, já discutidas.

\section{CONCLUSÕES}

1. Para tempos de contagem maiores que $4 \mathrm{~s}$, não existe variabilidade entre as leituras obtidas para cada tempo de contagem.

2. Existe grau de variabilidade dos dados de leituras dentro de seus respectivos tempos de contagem com o aumento de profundidade, evidenciando que a variabilidade espacial do solo em questão é um fator que não pode ser desprezado quando se utiliza sonda de nêutrons para determinar a umidade do solo.

3. Os tempos de contagem utilizados nas medições com a sonda de nêutrons tiveram importante influência na magnitude da densidade de nêutrons (leituras).

4. Repetições das leituras em um mesmo ponto podem ser dispensadas quando o tempo de contagem for igual ou superior a $32 \mathrm{~s}$.

5. Para se determinar o conteúdo de água do solo, recomenda-se fazer uma leitura em cada profundidade, aos $30 \mathrm{~s}$.

\section{LITERATURA CITADA}

Beltrame, L.; Taylor, J.C. Uso da sonda de nêutrons para determinação da umidade do solo no campo. Revista Brasileira de Ciência do Solo, Campinas, v.4, p.57-61, 1980.

Carneiro, C.; de Jong, E. In situ determination of the slope of the calibration curve of a neutron probe using a volumetric technique. Soil Science, Baltimore v.139, p.250-254, 1989.

Chawla, A.S.; Tripathi, S.K. Neutron moisture probe calibration for crop water management. In: International Conference on Measurement of Soil and Plant Water Status, 1987. Proceedings... Logan: Utah State University, v.1. 1987. p.29-35. Costa Neto, P.L.O. Estatística. 10 ed. São Paulo: Edgard Blucher, 1990.264p.

Encarnação, F.; Carneiro, C.; Dall'olio, A.; Vachaud, G. Técnica volumétrica de calibração de uma sonda de nêutrons. Pesquisa Agropecuária Brasileira, Brasília, 1991, v.26, n.7, p.1107-1112, 1991.

Falleiros, M.C.; Reichardt, K.; Bacchi, O.S.; Rodrigues, J.A. Calibração da sonda de nêutrons medida nas proximidades da superfície do solo. In: Congresso Brasileiro de Ciência do Solo, 10, 1994, Florianópolis. Anais... Florianópolis: SBCS, 1994, p.304.

Gardner, W.H. Water content. In: Methods of soil analysis. Part I: Physical and mineralogical methods. A. Klute (ed). Madisosn: ASA-SSSA. p.493-544. 1985. Agronomy Series, No. 9 
Gomes, F.P. Curso de estatística experimental. Piracicaba: ESALQ, 1987.467p.

Guerra, H.O.C. Física dos solos. 1 ed. Campina Grande, PB: UFPB, 2000. 173p.

Havercamp, R.; Vauclin, M.; Vachaud, G. Error analysis in estimating soil water content from neutron probe measurements: 1. Local standpoint. Soil Science, Baltimore, v.137, n.2, p.78-90, 1984.

Horiushi, S.; Nakeda, P. Nagayama, K. Moisture measurement for a radioactive waste neutron probe. Nuclear Instruments and Methods in Physics Research. Amsterdan, v.50, n.3, p.568-577, 2000 .

Marciano, M.C. Variabilidade das leituras de tensiômetros e sonda de nêutrons em experimentos de manejo de irrigação. Piracicaba: ESALQ, 1995. 149p. Dissertação Mestrado
Mishima. K.; Hibiki, T. Quantitative limits of thermal and fluid phenomena measurements using the neutron attenuation characteristics of materials. Experimental Thermal and Fluid Science, v.12, n.4, p.461-472, 1996.

Torres, P.S.; Gonzales, R. Determination of the humidity in representative soils of the western savannas using neutrons probes. In: Congreso Venezuelano Sobre la Ciencia del Suelo, 1993, Caracas, Venezuela. Programa y resúmenes de trabajos. 1993. p.75-76.

Wang, C.H.; Willis David L. Radiotracer methodology in biological science. Englewood Cliffs: Prentice-Hall. p.186-192. 1965. Biological Science Series 